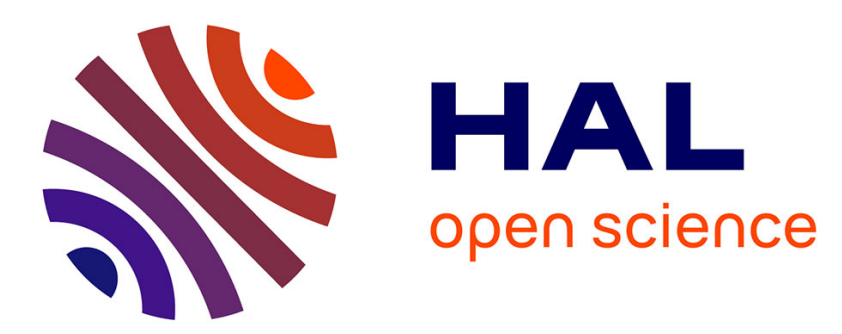

\title{
Simultaneous determination of thermal diffusivity and thermal conductivity of a thin layer using double modulated thermal excitations
}

Kamal Alaili, Jose Ordonez-Miranda, Younes Ezzahri

\section{To cite this version:}

Kamal Alaili, Jose Ordonez-Miranda, Younes Ezzahri. Simultaneous determination of thermal diffusivity and thermal conductivity of a thin layer using double modulated thermal excitations. Journal of Applied Physics, 2019, 126 (14), pp.145103. 10.1063/1.5116526 . hal-02321943

\section{HAL Id: hal-02321943 \\ https://hal.science/hal-02321943}

Submitted on 21 Nov 2020

HAL is a multi-disciplinary open access archive for the deposit and dissemination of scientific research documents, whether they are published or not. The documents may come from teaching and research institutions in France or abroad, or from public or private research centers.
L'archive ouverte pluridisciplinaire HAL, est destinée au dépôt et à la diffusion de documents scientifiques de niveau recherche, publiés ou non, émanant des établissements d'enseignement et de recherche français ou étrangers, des laboratoires publics ou privés. 


\title{
Simultaneous determination of thermal diffusivity and thermal conductivity of a thin layer using double modulated thermal excitations
}

\author{
Kamal Alaili, ${ }^{1, a)}$ Jose Ordonez-Miranda, ${ }^{1}$ and Younès Ezzahri ${ }^{1}$ \\ 1 Institut Pprime, CNRS-Université de Poitiers, ISAE-ENSMA, Futuroscope Chasseneuil F-86962, \\ France
}

(Dated: 17 October 2019)

A theoretical model is developed to determine simultaneously and in different ways thermal diffusivity and thermal conductivity of thin layers. This is done by using the accurate expression of the temperature distribution derived from the parabolic heat equation when the front surface of the thin layer is excited by a periodic heat flux, while the rear surface is maintained at one of three different types of boundary conditions; modulated periodic heat flux, modulated temperature, or constant temperature. Our approach exploits the modulation frequencies at which the normalized front surface temperature reaches its first maximum and first minimum. It is shown that (i) these characteristic frequencies can be used to obtain the thermal diffusivity of the finite layer under three different types of boundary conditions. (ii) the ratio between the values of the maxima and minima of the temperature can be utilized to determine the thermal conductivity of the finite layer. These two thermal properties are sensitive to the nature of the boundary conditions as well as the modulation frequency of the heat excitation. This paper provides a theoretical basis for the determination of the thermal diffusivity and thermal conductivity of the finite layer using laser-based heating photothermal techniques.

\section{INTRODUCTION}

Frequency-domain and time-domain photothermal methods have been extensively utilized to determine the thermal properties of materials by comparing the theoretical results to the measured experimental data. ${ }^{1-4}$ The principle of these spectroscopic methods is based on the measurement of the thermal profile at one surface of the sample, when the latter is illuminated by a laser beam of modulated intensity, which is optically absorbed and converted into heat at the surface of the sample. ${ }^{5}$ Two common photothermal techniques for detecting the signal are the photoacoustic spectrometry and infrared photothermal radiometry. ${ }^{6}$ Since the pioneering work of Rosencwaig and Gersho ${ }^{7}$ in thermal wave phenomena, significant efforts have been devoted to analyze the amplitude and phase of the photothermal signal in a wide variety of physical configurations. ${ }^{6,8,9}$ In general; the analysis of the photothermal signals may be complicated because they include the transfer function of the electronic instruments. ${ }^{6}$ In most applications, it is convenient to normalize the measured signal in order to eliminate the transfer function. The thermal properties are then obtained by fitting the experimental data to the appropriate theoretical models. A major application of photothermal experiments is related to measurement of the thermal diffusivity of materials. ${ }^{10-14}$ BalderasLopeza and Mandelis ${ }^{6}$ developed a self-normalization procedure in order to measure the thermal diffusivity of thin metallic layers using two photothermal methods: photoacoustic and radiometric detection. Based on this self-normalization technique, the authors showed that the

a) corresponding author : Kamal.alaili@univ-poitiers.fr corresponding measurement procedures involve linear fits in the photothermally thin and/or thick limits. By using the photothermal radiometry technique, Depriester et $a l .{ }^{15}$ proposed a method, which does not involve the multiparameter-fit to simultaneously obtain the thermal diffusivity and effusivity of solid materials. This was done by utilizing the ratio of photothermal radiometry signals obtained with and without the substrate. They used two characteristic modulation frequencies at which the normalized amplitude reaches its maximum value and the normalized phase passes through zero to determine the thermal diffusivity of vitreous carbon and lead-itanatezirconate ceramic samples. More recently, Vales-Pinzon et $a l .{ }^{16}$ have determined the thermal properties of the glassy carbon sample using four characteristic modulation frequencies in two-layer systems based on the analysis of the amplitude and phase of the photothermal signal. In this latter work, the authors showed that these four characteristic frequencies are independent of the thermal properties of the layers, and their values can be used to determine the thermal diffusivity and the thermal effusivity of the illuminated layer. Even though their results are in good agreement with those reported in the literature, ${ }^{6,15,16}$ their approach is however limited to the two-layer configuration. Therefore, the simultaneous determination of thermal diffusivity and thermal conductivity of a thin film layer is very desirable. Besides, in the case of periodic boundary conditions, an interesting phenomenon comes to play; the occurrence and propagation of thermal waves. Extensive efforts have been devoted to analyze the propagation processes of these thermal waves with analytical and numerical methods. ${ }^{17-22}$ In this regard, an interesting work was performed by Torii and Yang. ${ }^{17}$ The authors, also used numerical methods to study the effect of laser heating on the propagation phenomenon of a thermal wave in a very thin 
film subjected to a symmetrical heat excitation on both sides. Marin ${ }^{23}$ discussed the heat conduction in solids excited by a periodically time-dependent source, commonly encountered in photothermal experiments. Thermal wave transmission and reflection phenomena induced by a pulsed boundary heat flux in a two-layer slab with imperfect interface are studied numerically by Ramadan and Al-Nimr. ${ }^{24}$ Ordonez-Miranda and Alvarado-Gil ${ }^{18}$ investigated a one-dimensional thermal wave transport in a semi-infinite layer using the exact solution of dualphase-lag heat conduction model. Geiler et al. ${ }^{25}$ used double laser beams with different wave lengths in order to generate the differential frequency in the sample by photothermal modulation of the refractive index. In this context, Dietzel et al. ${ }^{26}$ developed the double modulated thermoreflectance microscopy of semi-conductor devices based on combined optical and electrical excitation. However, in these latter works, the problem of a semi-infinite layer has been studied extensively, and those with a finite layer do not offer analytical solutions. ${ }^{5,27} \mathrm{In}$ this paper, we solve analytically the parabolic heat equation (PHE) in order to determine the thermal diffusivity and thermal conductivity for a thin film layer, in which both sides are excited with periodic sources.

The purpose of this study is to analytically solve the PHE for the temperature profile in a finite layer, when both sides are excited by a modulated heat source. It is shown from the theoretical analysis of the photothermal signal that the characteristic frequencies at which the temperature of the front surface reaches its first maximum and minimum can easily be determined. It is also shown that these characteristic frequencies can be used to obtain the thermal diffusivity of the finite layer. Moreover, the ratio between the values of the maxima and minima of the temperature can be utilized to extract the thermal conductivity of the finite layer. Our approach is applied to a finite layer when the front surface is excited by a periodic heat flux, while the rear surface is maintained at one of three different types of boundary conditions; modulated periodic heat flux, modulated temperature, or constant temperature (see also the supplementary material). The paper is organized as follows: Sec. II presents the formulation of the physical problem using the PHE, while its key features are presented and discussed in Sec. III. We finish our work with summary and concluding remarks in Sec. IV.

\section{FORMULATION OF THE PROBLEM AND SOLUTIONS}

\section{A. Governing equation and physical problem}

Let us consider the configuration shown in Fig. 1, in which the layer system is confined between $x=0$ and $x=l$. We assume that the layer has a uniform thickness and constant thermophysical properties. Initially, the layer is at temperature $T(x, 0)=T_{0}$ which is the

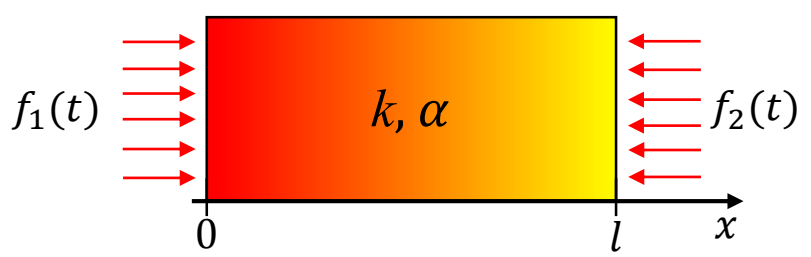

FIG. 1: Schematic diagram of the studied finite thickness layer of thermal conductivity $k$ and thermal diffusivity $\alpha$. The modulated thermal excitations are applied at $x=0$ and $x=l$.

ambient temperature. For time $t>0$, the boundaries at $x=0$ and $x=l$ are excited with modulated heat sources at frequencies $\omega_{1}$ and $\omega_{2}$, respectively. By assuming a one dimensional propagation through the layer, the thermal wave field at any position is given by:

$$
\frac{\partial T(x, t)}{\partial t}-\alpha \frac{\partial^{2} T(x, t)}{\partial x^{2}}=0,
$$

where $\alpha=k /\left(\rho c_{p}\right)$ is the thermal diffusivity, $k$ is thermal conductivity, $\rho$ is the density, and $c_{p}$ is the specific heat capacity of the material. For the sake of simplicity, we introduce the normalized position $z$, time $\tau$, temperature $\theta$, heat flux $Q$, and frequency $\Omega$, respectively, defined as:

$$
\begin{array}{r}
z=x / l, \quad \tau=t /\left(l^{2} / \alpha\right), \quad \theta=T / T_{0}, \quad Q=q /\left(k T_{0} / l\right), \\
\Omega=\omega /\left(\alpha / l^{2}\right) .
\end{array}
$$

After some simplifications, Eq. (1) can be written in a dimensionless form as:

$$
\frac{\partial \theta(z, \tau)}{\partial \tau}-\frac{\partial^{2} \theta(z, \tau)}{\partial z^{2}}=0 .
$$

\section{B. Boundary and initial conditions}

The boundary and initial conditions for the physical problem are:

$$
\begin{gathered}
\frac{\partial \theta(0, \tau)}{\partial z}=f_{1}(\tau)=-\frac{Q_{1}}{2}\left[1+\cos \left(\Omega_{1} \tau\right)\right] \\
\theta(z, 0)=1 .
\end{gathered}
$$

where $q_{1}=F \eta(1-R) q_{0}$, with $F$ being a parameter determined by the optical, thermal and geometric properties of the finite layer, ${ }^{28} \eta$ the efficiency at which the absorbed light is converted into heat, $R$ the reflection coefficient of the surface at $z=0$, and $q_{0}\left[\mathrm{Wm}^{-2}\right]$ the intensity of the laser beam. ${ }^{29-31}$ The surface $z=0$ is illuminated by a modulated heat flux, which is the case when the opaque surface of a medium is uniformly excited by a laser beam of modulated intensity. ${ }^{32}$ Furthermore, the opposite surface at $z=1$ considered in 
this work will be studied for three separate cases:

- Case 1: A modulated temperature imposed on the right-hand side surface

$$
\theta(1, \tau)=f_{2}(\tau)=1+m \cos \left(\Omega_{2} \tau\right)
$$

where $m$ is a positive constant. The modulated temperature (Eq. (5)) may be fulfilled when the medium at $z=1$ is in contact with a Peltier module producing an oscillating temperature. ${ }^{18,31}$ In practice, the temperature oscillations of a surface can be set with a Peltier cell, ${ }^{33}$ which typically operate with a resolution better than 0.1 $\mathrm{K}$ and modulation frequencies from 0.1 up to $100 \mathrm{~Hz}{ }^{34}$

- Case 2: A modulated heat flux applied on the righthand side surface

$$
\frac{\partial \theta(1, \tau)}{\partial z}=f_{2}(\tau)=\frac{Q_{2}}{2}\left[1+\cos \left(\Omega_{2} \tau\right)\right] .
$$

- Case 3: Two independent heat flux centered on two different frequencies $\Omega_{1}$ and $\Omega_{2}$ are applied at the surface $z=0$. This type of excitation was applied by Grégoire et al. ${ }^{35}$ to examine non-linear photothermal and photoacoustic phenomena. The opposite side at $z=1$, is maintained at a constant temperature.

$$
\frac{\partial \theta(0, \tau)}{\partial z}=f_{1}(\tau)=-\frac{Q_{1}}{2}\left[1+\cos \left(\Omega_{1} \tau\right)\right]-\frac{Q_{2}}{2}\left[1+\cos \left(\Omega_{2} \tau\right)\right]
$$

$$
\theta(1, \tau)=1
$$

The above physical problem is described by a heat conduction equation with nonhomogeneous boundary conditions. In order to determine the temperature distribution within the layer, we make use of the solution structure theorem. It is worth mentioning, that this theorem is applicable only in the case of heat conduction problems with homogeneous boundary conditions. ${ }^{19,36,37}$ Hence, to use this theorem, the homogeneous problem should first be established.

\section{Auxiliary homogeneous conditions}

As mentioned previously, the above mathematical problem includes nonhomogeneous boundary conditions that need to be converted into a problem with homogeneous boundary conditions such that the solution structure theorem can be applied to determine the temeprature distribution. We assume that $\theta$ has the following form:

$$
\theta(z, \tau)=v(z, \tau)+w(z, \tau)
$$

By using this transformation, the original problem can be expressed in a form which consists of a corresponding homogeneous problem with the function $v(z, \tau)$, and an auxiliary function, $w(z, \tau)$. Note that the auxiliary function $w(z, \tau)$ depends on the type of boundary conditions.
For more details, the readers are referred to reference [37]. In the present work, we will focus only on case 1; the other cases are detailed in the supplementary material.

According to Eqs. (4a) and (5), $w(z, \tau)$ is given by:

$$
w(z, \tau)=f_{1}(\tau)(z-1)+f_{2}(\tau) .
$$

By inserting Eq. (8) into Eqs. (3), (4a), (4b), and (5), we obtain the following system of equations:

$$
\left\{\begin{array}{l}
\frac{\partial v(z, \tau)}{\partial \tau}-\frac{\partial^{2} v(z, \tau)}{\partial z^{2}}=H(z, \tau) \\
\frac{\partial v(0, \tau)}{\partial z}=0 \\
v(1, \tau)=0 \\
v(z, 0)=f(z)
\end{array}\right.
$$

where

$$
\begin{array}{r}
H(z, \tau)=\frac{\partial^{2} w(z, \tau)}{\partial z^{2}}-\frac{\partial w(z, \tau)}{\partial \tau} \\
=-\frac{Q_{1}}{2} \Omega_{1} \sin \left(\Omega_{1} \tau\right)(z-1)+m \Omega_{2} \sin \left(\Omega_{2} \tau\right),
\end{array}
$$

and

$$
f(z)=\theta(z, 0)-w(z, 0)=Q_{1}(z-1)-m .
$$

Note that after the transformation procedure, the new function $H(z, \tau)$ appearing in Eq. (10a) plays a role similar to that of an internal heat source.

\section{Complete solution of the non-homogeneous problem}

The system of Eqs. (10a) - (10d) is homogeneous, and the solution can now be determined by utilizing the solution structure theorem. According to the superposition principle, we can write Eqs. (10a) - (10d) as two subproblems relatives to $v_{1}(z, \tau)$ and $v_{2}(z, \tau)$, respectively.

- Sub-problem 1: homogeneous equation and boundary conditions.

$$
\left\{\begin{array}{l}
\frac{\partial v_{1}(z, \tau)}{\partial \tau}-\frac{\partial^{2} v_{1}(z, \tau)}{\partial z^{2}}=0 \\
\frac{\partial v_{1}(0, \tau)}{\partial z}=0 \\
v_{1}(1, \tau)=0 \\
v_{1}(z, 0)=f(z)
\end{array}\right.
$$

- Sub-problem 2: inhomogeneous equation and homogeneous initial and boundary conditions.

$$
\left\{\begin{array}{l}
\frac{\partial v_{2}(z, \tau)}{\partial \tau}-\frac{\partial^{2} v_{2}(z, \tau)}{\partial z^{2}}=H(z, \tau) \\
\frac{\partial v_{2}(0, \tau)}{\partial z}=0 \\
v_{2}(1, \tau)=0 \\
v_{2}(z, 0)=0
\end{array}\right.
$$


By using the method of Fourier series, Eq. (13a) has the following solution:

$$
v_{1}(z, \tau)=\sum_{n=0}^{+\infty} v_{n}(\tau)\left[\cos \left(\lambda_{n} z\right)+\sin \left(\lambda_{n} z\right)\right]
$$

With the application of the first boundary condition (Eq. (13b)), Eq. (15) reduces to

$$
v_{1}(z, \tau)=\sum_{n=0}^{+\infty} v_{n}(\tau) \cos \left(\lambda_{n} z\right)
$$

By appling the second boundary condition (Eq. (13c)), the eigenvalues in Eq. (16) take the form:

$$
\lambda_{n}=(n+1 / 2) \pi \text {. }
$$

Substituting Eq. (16) into Eq. (13a) leads to

$$
\frac{d v_{n}(\tau)}{d \tau}+\lambda_{n}^{2} v_{n}(\tau)=0
$$

The solution of Eq. (18) is straightforward and is given by:

$$
v_{n}(\tau)=a_{n} e^{-\lambda_{n}^{2} \tau}
$$

Therefore, Eq. (16) can be expressed as

$$
v_{1}(z, \tau)=\sum_{n=0}^{+\infty} a_{n} e^{-\lambda_{n}^{2} \tau} \cos \left(\lambda_{n} z\right)
$$

The coefficients $a_{n}$ can be calculated by appling the initial condition (see Eq. (13d)). After some mathematical manipulations, we obtain:

$$
a_{n}=2 \int_{0}^{1} f(z) \cos \left(\lambda_{n} z\right) d z
$$

Inserting Eq. (12) into Eq. (21), leads to

$$
a_{n}=-\frac{2 Q_{1}}{(1 / 2+n)^{2} \pi^{2}}-\frac{2 m(-1)^{n}}{(1 / 2+n) \pi} .
$$

For the second sub-problem, $v_{2}(x, \tau)$, the solution can be obtained by using the solution structure theorem, ${ }^{37}$ which takes the form:

$$
v_{2}(z, \tau)=\int_{0}^{\tau} \int_{0}^{1} G\left(z, \xi, \tau-\tau^{\prime}\right) H\left(\xi, \tau^{\prime}\right) d \xi d \tau^{\prime},
$$

where $G\left(z, \xi, \tau-\tau^{\prime}\right)$ is the Green function, that is defined by:

$$
G\left(z, \xi, \tau-\tau^{\prime}\right)=\sum_{n=0}^{+\infty} 2 e^{-\lambda_{n}^{2}\left(\tau-\tau^{\prime}\right)} \cos \left(\lambda_{n} \xi\right) \cos \left(\lambda_{n} z\right)
$$

By combining Eqs. (11), (23), and (24), we get the following solution for the second sub-problem

$$
v_{2}(z, \tau)=\sum_{n=0}^{+\infty}\left[A_{n} \psi_{n, 1}(\tau)+B_{n} \psi_{n, 2}(\tau)\right] \cos \left(\lambda_{n} z\right),
$$

where

$$
\begin{gathered}
A_{n}=\frac{Q_{1}}{(1 / 2+n)^{2} \pi^{2}\left(1+\chi_{n, 1}^{2}\right)}, \\
B_{n}=\frac{2 m(-1)^{n}}{(1 / 2+n) \pi\left(1+\chi_{n, 2}^{2}\right)}, \\
\psi_{n, j}(\tau)=e^{-\lambda_{n}^{2} \tau}-\cos \left(\Omega_{j} \tau\right)+\chi_{n, j} \sin \left(\Omega_{j} \tau\right) \quad(j=1,2), \\
\chi_{n, j}=\frac{\lambda_{n}^{2}}{\Omega_{j}} .
\end{gathered}
$$

The complete temperature distribution inside the finite layer is thus the sum of Eqs. (9), (20), and (25), which leads to:

$$
\theta(z, \tau)=w(z, \tau)+v_{1}(z, \tau)+v_{2}(z, \tau)
$$

For any of the modulated thermal excitations, the temperature distribution at any point of the medium can be written as follows ${ }^{28}$

$$
\theta(z, \tau)=1+\theta_{\mathrm{dc}}(z)+\theta_{\mathrm{ac}}(z, \tau)
$$

where the unity corresponds to the ambient temperature, $\theta_{\mathrm{dc}}(z)$ and $\theta_{\mathrm{ac}}(z, \tau)$ are the stationary and periodic components of the temperature, respectively. These last two terms are associated to the first and second terms of the thermal source excitation, respectively. The combination of Eqs. (27) and (28) fully determines the expressions of $\theta_{\mathrm{dc}}(z)$ and $\theta_{\mathrm{ac}}(z, \tau)$. Equation (28) satisfies all the imposed boundary conditions along with the temperature differential equation and therefore it is the correct solution for the temperature profile, as established by the fundamental theorem of differential equations. ${ }^{38}$ In addition, in the limit $\Omega_{2}=0$ and $m=0$, Eq. (27) reduces to previous results reported by Rodney and Diana. ${ }^{39}$ Taking into account that the transient heat conduction is driven by both the thermal diffusivity and thermal effusivity (thermal conductivity), $6,16,18$ their simultaneous determination is therefore desirable.

\section{DETERMINATION OF THE THERMAL PROPERTIES}

In this section, the explicit expressions for the thermal diffusivity and thermal conductivity are obtained using the complete temperature distribution inside the material (Eq. (27)). Given that this temperature distribution oscillates in time with respect to the boundaries conditions (Eqs. (4a) and (5)), we calculate the average temperature over a period $\Gamma_{2}\left(\Gamma_{2}=2 \pi / \Omega_{2}\right)$. 
The average temperature over $\Gamma_{2}$ is given by:

$$
\bar{\theta}(z)=\frac{1}{\Gamma_{2}} \int_{0}^{\Gamma_{2}} \theta(z, \tau) d \tau .
$$

Even though most photothermal techniques use lockin amplifiers to measure the amplitude and phase of the temperature field averaged over many heating periods, ${ }^{6,15}$ the time variations of the physical temperature and its average value $\bar{\theta}$ can also be visualized and recorded through an oscilloscope, for different modulation frequencies, as reported by S. Dilhaire et al. ${ }^{40}$

After inserting Eq. (27) into Eq. (29), the following expression for $\bar{\theta}(z)$ is obtained

$$
\begin{gathered}
\bar{\theta}(z)=1-\frac{Q_{1}}{2}\left[1+\frac{\Omega_{2}}{2 \pi \Omega_{1}} \sin \left(\frac{2 \pi \Omega_{1}}{\Omega_{2}}\right)\right](z-1) \\
+\frac{\Omega_{2}}{2 \pi} \sum_{n=0}^{+\infty}\left\{\frac{a_{n}+A_{n}+B_{n}}{\lambda_{n}^{2}}+\frac{2 A_{n}}{\Omega_{1}} \sin \left(\frac{\pi \Omega_{1}}{\Omega_{2}}\right)\right. \\
\left.\cdot\left[\frac{\lambda_{n}^{2}}{\Omega_{1}} \sin \left(\frac{\pi \Omega_{1}}{\Omega_{2}}\right)-\cos \left(\frac{\pi \Omega_{1}}{\Omega_{2}}\right)\right]\right\} \cos \left(\lambda_{n} z\right) .
\end{gathered}
$$

As in the case of the temperature, the average heat flux over $\Gamma_{2}$ at the illuminated surface is straightforward and is given by:

$$
\bar{Q}=\frac{Q_{1}}{2}\left[1+\frac{\Omega_{2}}{2 \pi \Omega_{1}} \sin \left(\frac{2 \pi \Omega_{1}}{\Omega_{2}}\right)\right] .
$$

Note that both Eqs. (30) and (31) can be written as the sum of a steady state and modulated components. According to Eq. (30), the temperature field expression depends on the thermal diffusivity $\alpha$ and thermal conductivity $k$ of the material, and therefore this expression can be used to determine these thermal properties. The determination of $\alpha$ was already done by using one frequency modulated excitation source applied at the surface $z=0 .{ }^{15,16}$ In the present work, both thermal diffusivity and thermal conductivity are obtained based on the expression of the average temperature over a period $\Gamma_{2}$ (Eq. (30)). This is achieved by means of a meticulous graphical examination of the features of this average temperature at its first maximum and first minimum values. The normalized average temperature $\bar{\theta}$ and heat flux $\bar{Q}$ at the illuminated surface $z=0$ as functions of the normalized frequency $\Omega_{1}$ are shown in Figs. 2(a) - 2(b), respectively; for two values of $\Omega_{2}$. Note that the average temperature presents the typical behavior of traditional thermal wave phenomena for $\Omega_{2}=0.1$. When the modulation frequency increases $\left(\Omega_{1} \gg 1\right)$, the temperature oscillations manifest a strong attenuation. Furthermore, the penetration depth of the temperature oscillation (thermal wave) which depends on the modulation frequency of the heat source $\left(\mu_{1}=\sqrt{2 \alpha / \omega_{1}}\right)$ reduces as the excitation frequency increases. These results agree well with the the work reported by Torii and Yang ${ }^{17}$ and OrdonezMiranda et al. ${ }^{28}$. In the intermediate regime, the average
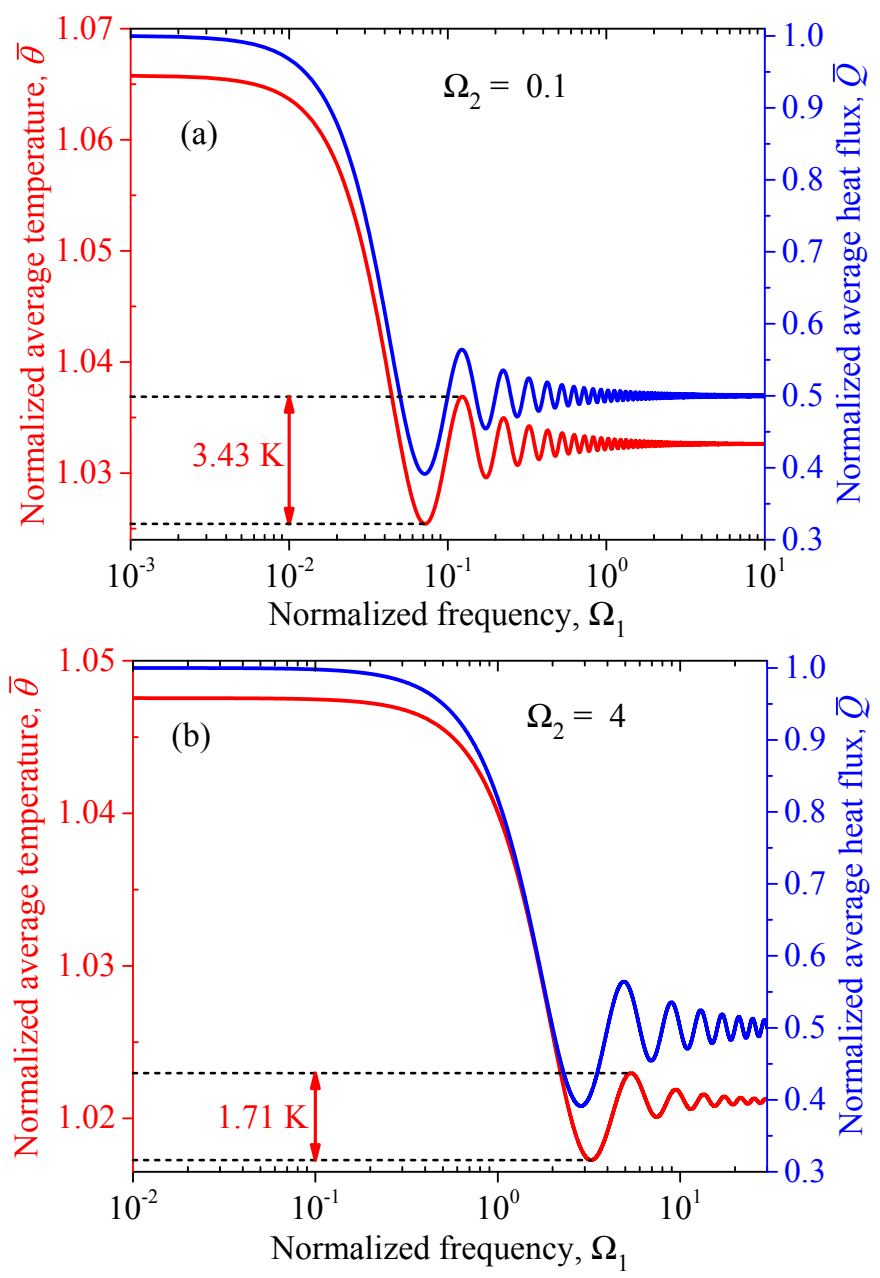

FIG. 2: Normalized average temperature and heat flux at the illuminated surface $z=0$ for Case 1 as functions of the normalized frequency $\Omega_{1}$ for (a) $\Omega_{2}=0.1$ and (b)

$\Omega_{2}=4$. The up down arrow corresponds to the

difference between the first minimum and first maximum of the temperature. Calculations have been made for $l=10^{-3} \mathrm{~m}, k=100 \mathrm{Wm}^{-1} \mathrm{~K}^{-1}, T_{0}=300 \mathrm{~K}$, $m=1 / 15$, and $q_{1}=2.10^{6} \mathrm{Wm}^{-2}$.

temperature distribution inside the thin layer oscillates in time and the values of its first maximum and first minimum decrease when $\Omega_{2}$ increases (see Figs. 2(a) - 2(b)). This was expected, with respect to Eqs. (4a) and (5). For low frequencies $\left(\Omega_{1} \ll 1\right)$, the behavior of the temperature and heat flux takes a constant value and becomes independent of the modulation frequency with respect to the steady-state conditions. The difference between the first maximum and first minimum of the temperature is respectively, $3.43 \mathrm{~K}$ and $1.71 \mathrm{~K}$ for $\Omega_{2}=0.1$ and $\Omega_{2}=4$. Experimentally, this difference is easily measurable with $\left(T_{1, \max }-T_{1, \min }\right) \geq 1 \mathrm{~K}$. We obtain similar results for the Case 2 and Case 3 . We provide more details in the supplementary material. According to complex the derivations in Sec. II, it is almost impossible to get analytically the expressions of the first maximum and 
minimum of the temperature as well as the frequencies at which they occur. The numerical solution of Eq. (30) makes it possible to calculate the frequencies at which the temperature reaches its first maximum and minimum, as shown in Fig. 3. In this figure, we plot the frequencies at which the temperature reaches its first maximum and minimum as functions of the normalized frequency $\Omega_{2}$. We note these frequencies $\Omega_{1, \max }$ and $\Omega_{1, \min }$, respectively. These frequencies increase linearly as functions of $\Omega_{2}$ which is consistent with the results shown in Figs. 2(a)- 2(b). Furthermore, the relationship between $\Omega_{1}$ and $\Omega_{2}$, where the normalized average temperature reaches its first maximum and first minimum values can be obtained through fitting. Given that for small values of $\Omega_{2}\left(0.001 \leq \Omega_{2}<1\right), \Omega_{1, \max }$ and $\Omega_{1, \min }$ varies linearly with $\Omega_{2}$ (see Fig. 3 (a)). The results obtained through fitting lead to $\Omega_{1, \max } \approx(9 / 7) \Omega_{2}$ and $\Omega_{1, \min } \approx(3 / 4) \Omega_{2}$. Therfore, the proposed method based on subtracting the minimum and maximum frequencies cannot be used to determine $\alpha$. By contrast, in the high frequency regime $\left(\Omega_{2}>1\right)$, the first minimum and maximum of $\bar{\theta}$ occur for a linear dependence between $\Omega_{1}$ and $\Omega_{2}$ (see Fig. 3 (b)) and we can write

$$
\begin{cases}\Omega_{1, \min } \approx \frac{9}{11} \Omega_{2}-\frac{1}{17}, & \bar{\theta}=\bar{\theta}_{1, \min } \\ \Omega_{1, \max } \approx \frac{15}{11} \Omega_{2}-\frac{2}{35}, & \bar{\theta}=\bar{\theta}_{1, \max }\end{cases}
$$

where $\Omega_{j}=l^{2} \omega_{j} / \alpha$.

Given that the Eqs. (32a) and (32b) are derived for the modulated components of the applied heat flux and temperature in Eqs. (4a) and (5), they are valid for high frequency regime $\left(\Omega_{2}>1\right)$. Note that Eqs. (32a) and (32b) are independent of the particular values of $m$ and $Q_{1}$. The variation of these parameters changes the temperature $\bar{\theta}_{1, \max }$ and $\bar{\theta}_{1, \min }$ but not the modulation frequencies of their minima and maxima. One can obtain the thermal diffusivity of the sample by combining Eqs. (32a) and (32b), as follows:

$$
\alpha \approx \pi l^{2}\left(48.9 f_{1, \max }-81.5 f_{1, \min }\right),
$$

where $\omega_{j}=2 \pi f_{j}$. Therefore, Eq. (33) will lead to obtaining the thermal diffusivity of the sample, by performing a simple frequency scan to determine the modulation frequencies where the average temperature at the illuminated surface $z=0$ reaches its first minimum and maximum values. According to Eq. (33), the thermal diffusivity $\alpha$ is highly sensitive to the frequencies $f_{1, \max }$ and $f_{1, \text { min }}$ at which the maximum and minimum values of the temperature field occur, respectively. In practice, the accurate determination of $\alpha$ will thus need to repeat the required experiments a number of times to measure representative values of these two frequencies together with their uncertainties.

According to Eq. (30), the average temperature over a period $\Gamma_{2}$ at the illuminated surface $z=0$ can be conveniently rewritten as follows:

$$
\bar{\theta}(0)=1+Q_{1} F_{1}\left(\Omega_{1}, \Omega_{2}\right)+F_{2}\left(\Omega_{2}\right),
$$
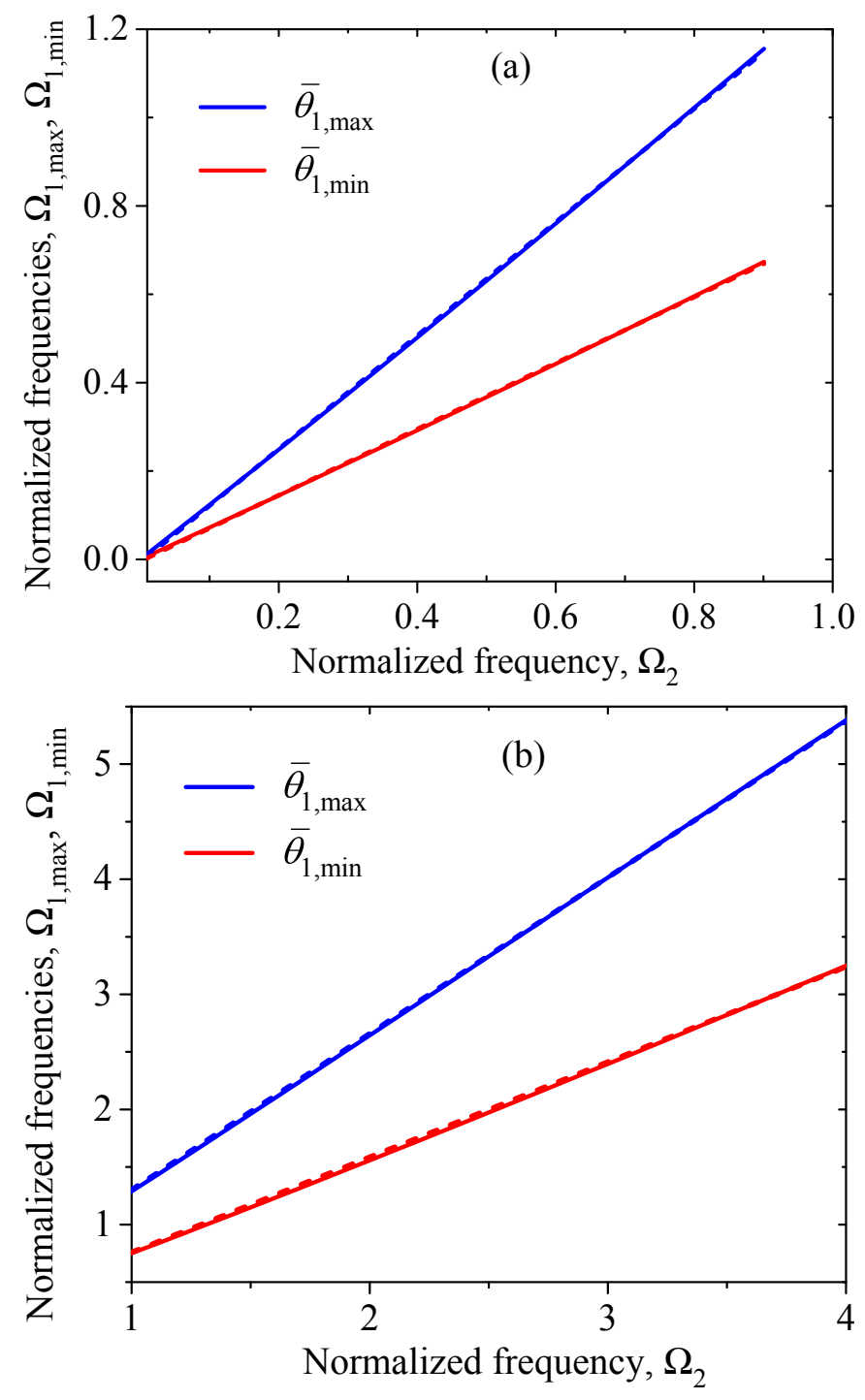

FIG. 3: Behavior of $\Omega_{1, \max }$ and $\Omega_{1, \min }$ as functions of $\Omega_{2}$ for Case 1. Calculations have been made for $l=10^{-3} \mathrm{~m}, k=100 \mathrm{Wm}^{-1} \mathrm{~K}^{-1}, T_{0}=300 \mathrm{~K}, m=1 / 15$, and $q_{1}=2.10^{6} \mathrm{Wm}^{-2}$. Dashed lines correspond to the linear fit and solid one correspond to the numerical solution of Eq. (30).

where

$$
\begin{aligned}
& F_{1}\left(\Omega_{1}, \Omega_{2}\right)= \frac{1}{2}\left[1+\frac{\Omega_{2}}{2 \pi \Omega_{1}} \sin \left(\frac{2 \pi \Omega_{1}}{\Omega_{2}}\right)\right] \\
&+\frac{\Omega_{2}}{2 \pi} \sum_{n=0}^{+\infty}\left\{\frac{a_{n}^{\prime}+A_{n}^{\prime}}{\lambda_{n}^{2}}+\frac{2 A_{n}^{\prime}}{\Omega_{1}} \sin \left(\frac{\pi \Omega_{1}}{\Omega_{2}}\right)\right. \\
&\left.\cdot\left[\frac{\lambda_{n}^{2}}{\Omega_{1}} \sin \left(\frac{\pi \Omega_{1}}{\Omega_{2}}\right)-\cos \left(\frac{\pi \Omega_{1}}{\Omega_{2}}\right)\right]\right\},
\end{aligned}
$$




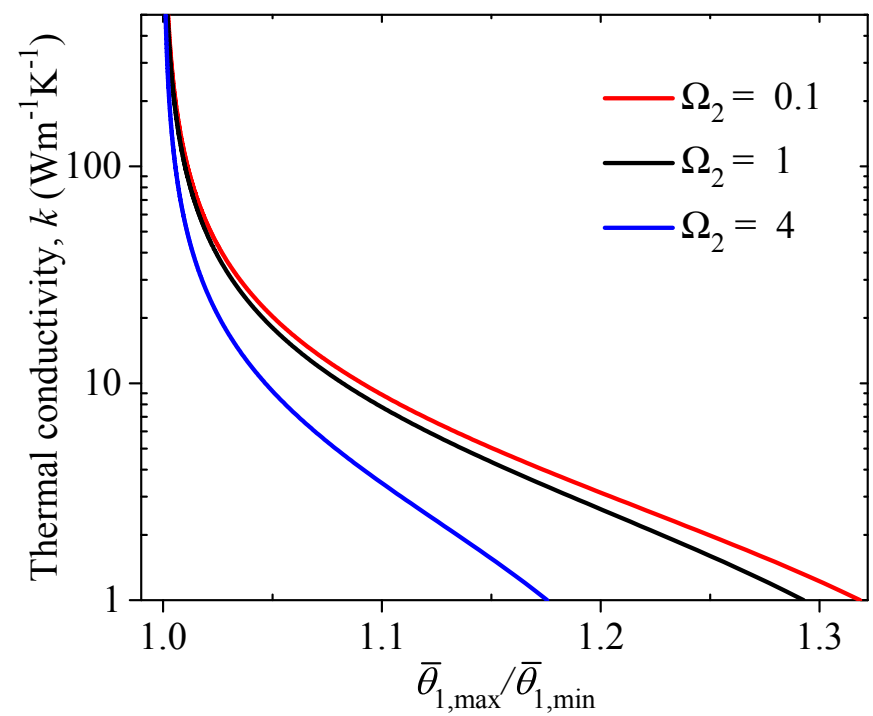

FIG. 4: The computed behavior of the ratio $\left(\bar{\theta}_{1, \max } / \bar{\theta}_{1, \min }\right)$ between the first maximum and minimum values of the temperature for Case 1 as a function of thermal conductivity $k$ for three values of

$\Omega_{2}$. Calculations have been made for $l=10^{-3} \mathrm{~m}$, $T_{0}=300 \mathrm{~K}, m=1 / 15$, and $q_{1}=2.10^{6} \mathrm{Wm}^{-2}$.

and

$$
\left\{\begin{array}{c}
F_{2}\left(\Omega_{2}\right)=\frac{\Omega_{2}}{2 \pi} \sum_{n=0}^{+\infty} \frac{1}{\lambda_{n}^{2}}\left[B_{n}-\frac{2 m(-1)^{n}}{(1 / 2+n) \pi}\right], \\
a_{n}^{\prime}=-\frac{2}{(1 / 2+n)^{2} \pi^{2}} \text { and } A_{n}^{\prime}=A_{n} / Q_{1} .
\end{array}\right.
$$

Given that the detected photothermal signal is proportional to the modulated temperature defined in Eq. (34), it is convenient to normalize the measured maximum signal by the minimum one. By doing so, we can write:

$$
\frac{\bar{\theta}_{1, \max }}{\bar{\theta}_{1, \text { min }}}=\frac{1+Q_{1} F_{1}\left(\Omega_{1, \max }, \Omega_{2}\right)+F_{2}\left(\Omega_{2}\right)}{1+Q_{1} F_{1}\left(\Omega_{1, \min }, \Omega_{2}\right)+F_{2}\left(\Omega_{2}\right)},
$$

where $\Omega_{1, \min }$ and $\Omega_{1, \max }$ are related to $\Omega_{2}$ by Eqs. (32a) and (32b), respectively. After simplifications, Eq. (37) yields

$k=\frac{q_{1} l l}{T_{0}} \frac{F_{1}\left(\Omega_{1, \max }, \Omega_{2}\right)-\left(\bar{\theta}_{1, \max } / \bar{\theta}_{1, \min }\right) F_{1}\left(\Omega_{1, \min }, \Omega_{2}\right)}{\left(\left(\bar{\theta}_{1, \max } / \bar{\theta}_{1, \min }\right)-1\right)\left(1+F_{2}\left(\Omega_{2}\right)\right)}$.

Note that $k$ depends on the ratio $\left(\bar{\theta}_{1, \max } / \bar{\theta}_{1, \text { min }}\right)$ and the absorbed heat flux $q_{1}$, which can be considered nearly constant and independent of the modulation frequency, as is usually done in similar problems. ${ }^{29,31}$ Equations (33) and (38) indicate that the simultaneous determination of both the thermal diffusivity and thermal conductivity of a layer only requires the measurement of two characteristic frequencies of the temperature signal. This fact represents one of the advantages of the proposed method with respect to other photothermal ones, such as thermoreflectance, which requires recording the amplitude and phase of the temperature field over wide intervals of modulation frequencies to retrieve the same properties. ${ }^{41}$

The determination of $\Omega_{1, \max }$ and $\Omega_{1, \min }$ and their corresponding temperature magnitudes $\bar{\theta}_{1, \max }$ and $\bar{\theta}_{1, \text { min }}$ allows therefore the extraction of the thermal conductivity $k$ of the thin film layer trough application of Eq. (38). The precision of $k$ will depend on the precision of these input parameters as well as the number of terms considered in functions $F_{1}$ and $F_{2}$. We report in Fig. 4 the computed behavior of the thermal conductivity $k$ as a function of the ratio between the first maximum and first minimum values of the temperature $\left(\bar{\theta}_{1, \max } / \bar{\theta}_{1, \min }\right)$. Note that $k$ depends strongly on the ratio $\left(\bar{\theta}_{1, \max } / \bar{\theta}_{1, \text { min }}\right)$ as well as the normalized frequency $\Omega_{2} . \quad k$ decreases as the ratio $\left(\bar{\theta}_{1, \max } / \bar{\theta}_{1, \min }\right)$ increases and when the latter approaches unity, $k$ tends to infinity. We notice also that the ratio $\left(\bar{\theta}_{1, \max } / \bar{\theta}_{1, \min }\right)$ takes higher values for low frequencies $\Omega_{2}$; the determination of the thermal conductivity $k$ becomes more precise.

Taking into account that the Biot number $B i=h l / k$ represents the fraction of the thermal resistance $(l / k)$ of the material that opposes the heat convection characterized by the heat transfer coefficien $h$, the convective losses are expected to be negligible for good thermal conductors $B i \ll 1$ excited with relatively high modulation frequencies, as demonstrated by Martinez et al. ${ }^{42}$ In addition, given that the proposed method involves temperature gradients of a few degrees only (see Fig. 2), the radiation effects can also be neglected during the realization of the required experiments, as established by the Stefan-Boltzmann law of heat radiation. Finally, it is worthwhile to mention that even though all our results have been obtained for a heating with a sinusoidal profile, the developed analytical methodology is also expected to be valid for other profiles, as is the case of the squarewave one that is commonly used in chopped experiments.

\section{CONCLUSION}

A simple method for determining simultaneously the thermal diffusivity and thermal conductivity of a thin layer has been developed, based on the analytical solution of the one-dimensional parabolic heat equation. This is achieved by using the exact expression of the normalized temperature at the illuminated front surface of a thin layer when a periodic heat flux is applied at this surface, while the rear surface is maintained at one of three different types of boundary conditions; modulated heat flux, modulated temperature, or constant temperature. We have determined the frequencies at which the normalized surface temperature reaches its first 
maximum and first minimum. It has been demonstrated that these characteristic frequencies can be used to determine the thermal diffusivity of the finite layer. Furthermore, the combination of these frequencies and their corresponding temperature magnitudes, can easily lead to the determination of the thermal conductivity of the thin layer. Once the thermal diffusivity and thermal conductivity are known, one could extract the specific heat per unit volume. Therefore, application of the suggested method to a single photothermal experiment would allow determination of a set of three thermal properties of the thin layer.

\section{SUPPLEMENTARY MATERIAL}

For more details are provided in the supplementary material.

${ }^{1}$ W. B. Jackson, N. M. Amer, A. Boccara, and D. Fournier, Appl. Opt. 20, 1333 (1981).

${ }^{2}$ A. Boccara, D. Fournier, and J. Badoz, Appl. Phys. Lett. 36, 130 (1980).

${ }^{3}$ P. Hess and J. Pelzl, Photoacoustic and Photothermal Phenomena: Proceedings of the 5th International Topical Meeting, Heidelberg, Fed. Rep. of Germany, July 27-30, 1987, Vol. 58 (Springer, 1988).

${ }^{4}$ J. Ordonez-Miranda and J. Alvarado-Gil, J. Appl. Phys. 112, 114902 (2012).

${ }^{5}$ A. Mandelis, Diffusion-wave fields: mathematical methods and Green functions (Springer Science \& Business Media, 2001).

${ }^{6}$ J. A. Balderas-Lopez and A. Mandelis, Rev. Sci. Instrum. 74, 5219 (2003).

${ }^{7}$ A. Rosencwaig and A. Gersho, J. Appl. Phys. 47, 64 (1976).

${ }^{8}$ A. Mandelis and P. Hess, Semiconductors and electronic materials, Vol. 4 (Spie Press, 2000).

${ }^{9}$ A. Salazar, R. Fuente, E. Apinaniz, A. Mendioroz, and R. Celorrio, J. Appl. Phys. 110, 033516 (2011).

${ }^{10}$ G. Rousset, F. Lepoutre, and L. Bertrand, J. Appl. Phys. 54, 2383 (1983).

${ }^{11}$ L. Perondi and L. Miranda, J. Appl. Phys. 62, 2955 (1987).

${ }^{12}$ J. A. Balderas-Lopez, S. Tomas, H. Vargas, V. Olalde-Portugal, et al., Forest Prod. J. 46, 84 (1996).

${ }^{13}$ J. A. Balderas-Lopez and A. Mandelis, J. Appl. Phys. 90, 2273 (2001).

${ }^{14}$ O. Pessoa Jr, C. Cesar, N. Patel, H. Vargas, C. Ghizoni, and L. Miranda, J. Appl. Phys. 59, 1316 (1986).

${ }^{15}$ M. Depriester, P. Hus, S. Delenclos, and A. H. Sahraoui, Rev. Sci. Instrum. 76, 074902 (2005).

${ }^{16}$ C. Vales-Pinzon, J. Ordonez-Miranda, and J. Alvarado-Gil, J. Appl. Phys. 112, 064909 (2012).
${ }^{17}$ S. Torii and W.-J. Yang, Int. J. Heat Mass Transfer 48, 537 (2005).

${ }^{18}$ J. Ordóñez-Miranda and J. Alvarado-Gil, Mech. Res. Commun. 37, 276 (2010).

${ }^{19}$ T. T. Lam and E. Fong, Int. J. Therm. Sci. 50, 2104 (2011).

${ }^{20}$ M.-K. Zhang, B.-Y. Cao, and Y.-C. Guo, Int. J. Heat Mass Transfer 67, 1072 (2013).

${ }^{21}$ T. T. Lam, Int. J. Therm. Sci. 49, 1639 (2010).

${ }^{22}$ E. Fong and T. T. Lam, Int. J. Therm. Sci. 77, 55 (2014).

${ }^{23}$ E. Marín, J. Marín-Antuña, and P. Díaz-Arencibia, Eur. J. Phys. 23, 523 (2002).

${ }^{24}$ K. Ramadan and M. A. Al-Nimr, Heat Transfer Eng. 30, 677 (2009).

${ }^{25}$ H. Geiler, M. Wagner, P. Kowalski, and R. Bleiß, in Photoacoustic and Photothermal Phenomena III (Springer, 1992) pp. 477-481.

${ }^{26}$ D. Dietzel, B. Bein, and J. Pelzl, J. Appl. phys. 93, 9043 (2003).

${ }^{27} \mathrm{~W}$. Ang, Southeast Asian Bulletin of Mathematics 26, 185 (2003).

${ }^{28}$ J. Ordonez-Miranda and J. Alvarado-Gil, Int. J. Therm. Sci. 48, 2053 (2009).

${ }^{29}$ S. Galović and D. Kostoski, J. Appl. Phys. 93, 3063 (2003).

${ }^{30}$ A. Salazar, A. Sanchez-Lavega, and J. Terron, J. Appl. phys. 84, 3031 (1998).

${ }^{31}$ D. P. Almond, P. Patel, and P. Patel, Photothermal science and techniques, Vol. 10 (Springer Science \& Business Media, 1996).

32 J. Ordonez-Miranda and J. Alvarado-Gil, Int. J. Therm. Sci. 49, 209 (2010).

${ }^{33}$ J. Bodzenta, B. Burak, M. Nowak, M. Pyka, M. Szałajko, and M. Tanasiewicz, Dent. Mater. J. 22, 617 (2006).

${ }^{34}$ F. Cervantes-Alvarez, J. Macias, and J. Alvarado-Gil, J. Phys. D. 51, 065302 (2018).

${ }^{35}$ G. Grégoire, V. Tournat, D. Mounier, and V. Gusev, Eur. Phys. J . Spec. Top. 153, 313 (2008).

${ }^{36}$ T. T. Lam and E. Fong, Heat Mass Transfer 49, 509 (2013).

${ }^{37} \mathrm{~L}$. Wang, X. Zhou, and X. Wei, Heat conduction: mathematical models and analytical solutions (Springer Science \& Business Media, 2007).

${ }^{38}$ M. R. Spiegel, "Applied differential equations," Tech. Rep. (1967).

${ }^{39}$ R. Hechavarría and D. Coello-Fiallos, in AIP Conference Proceedings, Vol. 2003 (AIP Publishing, 2018) p. 020007.

${ }^{40}$ S. Dilhaire, G. Pernot, G. Calbris, J.-M. Rampnoux, and S. Grauby, J. Appl. phys. 110, 114314 (2011).

${ }^{41}$ A. J. Schmidt, X. Chen, and G. Chen, Rev. Sci. Instrum. 79, 114902 (2008).

${ }^{42}$ K. Martínez, E. Marín, C. Glorieux, A. Lara-Bernal, A. Calderón, G. P. Rodríguez, and R. Ivanov, Int. J. Therm. Sci. 98, 202 (2015). 